\title{
SResarch Square
}

\section{Joint Effect of 25-hydroxyvitamin D and Secondhand Smoke Exposure on Hypertension in Non-smoking Women of Childbearing Age: NHANES 2007-2014}

\section{Qianqian Shen}

Qingdao University

\section{Qian Xu}

Qingdao University Affiliated Qingdao Women and Childrens Hospital: Qingdao Women and Children's Hospital

\section{Guoju Li}

Qingdao University Affiliated Qingdao Women and Childrens Hospital: Qingdao Women and Children's Hospital

\section{Lisheng Ren}

The Affiliated Hospital of Qingdao University

\section{Zhenhong Zhang \\ Qingdao University \\ Yangting Zhang \\ Qingdao University \\ Zhaoyi Zhong \\ Qingdao University \\ Xiaona Li \\ Qingdao University}

Qiuzhen Wang ( $\nabla$ qdwangqiuzhen@126.com )

Qingdao University https://orcid.org/0000-0001-5946-3884

\section{Research Article}

Keywords: 25-hydroxyvitamin D, secondhand smoke, hypertension, women of childbearing age, interaction

Posted Date: August 13th, 2021

DOI: https://doi.org/10.21203/rs.3.rs-796119/v1

License: (a) (i) This work is licensed under a Creative Commons Attribution 4.0 International License. Read Full License 
Version of Record: A version of this preprint was published at Environmental Health on November 15th, 2021. See the published version at https://doi.org/10.1186/s12940-021-00803-1. 


\section{Abstract}

\section{Background}

Vitamin D deficiency (VDD) may increase the risk for hypertension in women of childbearing age, and they may expose to secondhand smoke exposure (SHS) simultaneously. Till now, few studies have investigated the joint effects of VDD and SHS. We evaluated whether exposure to SHS modified the association between VDD and hypertension.

\section{Methods}

Data from 2007-2014 National Health and Nutrition Examination Surveys (NHANES) were analyzed. Our research subjects were 2802 nonsmoking and nonpregnant women of childbearing age (20-44 years old). Hypertension was defined based either on systolic blood pressure (SBP) $\geq 130 \mathrm{mmHg}$ and/or diastolic blood pressure $(\mathrm{DBP}) \geq 80 \mathrm{mmHg}$ or on now taking prescribed medicine for hypertension. The interactive effect of VDD and SHS on hypertension was evaluated by using logistic regression models, followed by strata-specific analyses.

Results

The prevalence of VDD in the hypertension group was significantly higher than that in the non-hypertension group ( $48.2 \%$ vs $40.7 \%, P=0.005)$, as well as the exposure rate of SHS $(35.7 \%$ vs $30.5 \%, P=0.017)$. VDD was positively associated with hypertension [adjusted odds ratio (aOR): $1.47,95 \%$ confidence interval (CI): $1.03-$ 2.08]. There was no association between secondhand smoke exposure and hypertension (aOR: $1.10,95 \% \mathrm{Cl}$ : 0.85-1.43). However, when exposed to SHS and VDD simultaneously, the aOR estimation of the risk of hypertension increased to $1.83(1.17,2.87)$ with a significant interactive effect between SHS and VDD

$\left(P_{\text {adjusted }}=0.009\right)$. When stratified according to $\mathrm{BMI}$ and race, the interactive effect was about twice the effect of VDD on hypertension alone in women who were overweight (for VDD: aOR $=2.37,95 \% \mathrm{Cl}$ : 1.05-5.47; for interaction: $\mathrm{aOR}=4.14,95 \% \mathrm{Cl}: 1.48-11.58)$, and a similar pattern could also be observed in Non-Hispanic Black (for VDD: $\mathrm{aOR}=2.74,95 \% \mathrm{Cl}: 1.11-6.73$; for interaction: $\mathrm{aOR}=4.60,95 \% \mathrm{Cl}: 1.53-13.80$ ).

\section{Conclusion}

There exist synergistic effects of SHS and VDD on the prevalence of hypertension in American women of childbearing age, with more significant effects in overweight women and Non-Hispanic Black women. Further studies are warranted to verify in other populations, and the molecular mechanisms underlying the joint effect of SHS and VDD need to be elucidated.

\section{Introduction}

Hypertension is an important worldwide public health challenge on account of its high morbidity and related cardiovascular risk ${ }^{1}$. The global burden related to high blood pressure (BP) has experienced a steady increase from $17.3 \%$ in 1990 to $20.5 \%$ in 2015 and is still increasing ${ }^{2,3}$. Parallel to the rising global incidence of hypertension, the prevalence of hypertension in women of childbearing age is constantly increasing. 
Hypertension in this population is the prominent risk factor of pregnancy-induced hypertension, such as preeclampsia and eclampsia, which are leading causes of maternal and fetal mortality. It is also associated with an increased risk of childhood adverse health outcomes in the long-term, including asthma ${ }^{4}$, elevated blood pressure ${ }^{5}$, as well as some rare childhood cancers ${ }^{6}$. Furthermore, women's unique physiological conditions can affect BP in several hormonal ways ${ }^{7}$, which also provides a unique risk for hypertensionrelated cardiovascular risk. Given the high prevalence and the important impact of hypertension in women of childbearing age on gestational hypertension, postmenopausal hypertension, identification of modifiable risk factors to prevent the development of hypertension is imperative.

Major risk factors of hypertension have been identified, such as genetic predisposition, diet, physical activity, and alcohol consumption ${ }^{8}$. Unlike non-modifiable risk factors, nutritional deficits and environmental factors are potentially modifiable and preventable causes. Vitamin D deficiency (VDD) is one of the most common nutritional deficiencies all over the world, with a prevalence of about 33\% in American pregnant/lactating women ${ }^{9}$. Although a growing body of evidence suggests that VDD may adversely affect women's blood pressure $^{10-12}$, there is still inconsistency in the literature ${ }^{13}$. Studies have demonstrated that smoking may trigger VDD ${ }^{14}$, which may potentially cause further hypertension; and secondhand smoke (SHS) may have a similar effect that it may be involved in the relationship between VD and hypertension.

While cigarette smoking is on the decline in the US, in 2019 smoking prevalence was still $14.0 \%{ }^{15}$. Data from a nationally representative sample suggests that up to $23.6 \%$ of females are exposed to SHS, as indicated by serum cotinine levels $>0.05 \mathrm{ng} / \mathrm{mL}^{16}$. To date, there have been several studies suggesting a significant positive association between SHS and hypertension ${ }^{17-19}$. Exposure to SHS is a common risk factor for hypertension.

Both VDD and SHS possess characteristics related to chronic inflammation or structural and functional alterations on arterial ${ }^{19,20}$. In addition, exposure to SHS may lead to VDD, which may further aggravate endothelial dysfunction, increase arterial stiffness and lead to hypertension. However, the effect of exposure to SHS together with the vitamin D status on hypertension has not been evaluated. Thus, taken together, we hypothesized that there is an interaction effect between vitamin D status and SHS on HBP in women.

Hence, we evaluated the interaction between vitamin D levels and exposure to SHS on the prevalence of hypertension among 20-44 years old women using data from the National Health and Nutrition Examination Survey (NHANES) 2007-2014. Knowledge of the association could help health authorities in decision-making regarding policies for health promotion and intervention to prevent hypertension in women of childbearing age.

\section{Methods}

\section{Study design}

NHANES is conducted biennially by the Centers for Disease Control and Prevention's National Center for Health Statistics (NCHS, a part of the Centers for Disease Control and Prevention) since 1999, which is a cross- 
sectional, complex multistage, nationally representative survey that assesses population health and nutritional status that is representative of the noninstitutionalized U.S. civilian population. The questionnaire data, physical examination data, and biospecimens from participants were collected. The NCHS Research Ethics Review Board reviewed and approved the study, and informed written consent was obtained from all participants before they took part in the study.

Only 4 cycles $(2007-2014)(\mathrm{N}=40417)$ were included in the present analysis due to the detailed serum $25(\mathrm{OH}) \mathrm{D}$ and cotinine data. Exclusion criteria included male participants $(\mathrm{n}=20180)$; female participants less than 20 years old $(n=8369)$ or older than 44 years old $(n=6938)$; self-reported a history of active smoking $(n$ $=1656$ ) and serum cotinine $\geq 10 \mathrm{ng} / \mathrm{mL}$ (the cut-off point for active smoking) $(\mathrm{n}=103)$; ;issing data for $25(\mathrm{OH}) D(n=380)$ and cotinine $(n=9) \square$ pregnant women $(n=149) \square$ abnormal energy intake (energy intake > $5000 \mathrm{kcal}$ or $<500 \mathrm{kcal}, \mathrm{n}=31$ ). Our analysis was restricted to the 2802 women of childbearing age, defined as 20-44 years old in NHANES. The flow of participant inclusion is displayed in Fig. 1.

\section{Definition of Hypertension}

Blood pressure measurements were taken during the NHANES examination visit. After resting quietly in a seated position for 5 minutes, blood pressure was measured three times using a mercury sphygmomanometer by well-trained examiners. A fourth reading was measured if required. Then, the means of systolic blood pressure (SBP) and diastolic blood pressure (DBP) were calculated.

According to the 2017 American College of Cardiology/American Heart Association (ACC/AHA) Guideline for the Prevention, Detection, Evaluation, and Management of High Blood Pressure in Adults ${ }^{8}$, Hypertension was defined as $\mathrm{SBP} \geq 130 \mathrm{mmHg}$ and(or) $\mathrm{DBP} \geq 80 \mathrm{mmHg}$. At the same time, participants who answered yes to the question: 'Are you now taking prescribed medicine for high BP?' were also defined as having hypertension.

\section{Serum 25-hydroxyvitamin D measurement}

Serum 25-hydroxyvitamin D [25(OH)D] is the widely accepted indicator of vitamin D nutritional status. Blood samples were collected from each participant woman at a mobile examination center (MEC) and were immediately frozen at $-30^{\circ} \mathrm{C}$ for measurement of serum $25(\mathrm{OH}) \mathrm{D}$ concentrations ${ }^{12}$. It measured by standardized liquid chromatography-tandem mass spectrometry (LC-MS/MS) or expressed as LC-MS/MS equivalent, depending on NHANES campaigns ${ }^{21}$. Vitamin D was treated as both continuous (Median and IQR, $\mathrm{nmol} / \mathrm{l})$ and categorical variable [sufficiency $(\geq 75 \mathrm{nmol} / \mathrm{l})$, insufficiency $(50-74.9 \mathrm{nmol} / \mathrm{l})$, and deficiency $(<50$ $\mathrm{nmol} / \mathrm{I})$ ] as suggested by $10 \mathrm{M}^{22}$.

\section{Secondhand smoke exposure}

In our study, current smokers and former smokers were distinguished from nonusers of tobacco products through a combination of self-reporting questionnaires and serum cotinine data ${ }^{23}$. Specifically, based on the answers to the questions SMQ020: "Have you smoked at least 100 cigarettes during your life?" and SMQ040: "Do you now smoke cigarettes?", the self-reported active, former, or never smoking behavior was confirmed ${ }^{24}$. After excluding people who have reported a history of smoking,participants were excluded if their serum cotinine concentrations greater than $10 \mathrm{ng} / \mathrm{mL}$ (the serum cotinine cutoff value of active smoking $\left.{ }^{25,26}\right)(\mathrm{n}=$ 103). After exclusion, the rest are non-smokers. Based on the previous studies ${ }^{26,27}$, non-smoking adults who 
had a serum cotinine concentration ranging from 0.05 to $10 \mathrm{ng} / \mathrm{mL}$ were regarded as exposed to SHS, and those whose serum cotinine concentration less than $0.05 \mathrm{ng} / \mathrm{ml}$ were regarded as no secondhand smoke exposure. Serum cotinine was measured by an isotope-dilution high-performance liquid chromatography/atmospheric pressure chemical ionization tandem mass spectrometric (ID HPLC-APCI MS/MS) method ${ }^{28}$.

\section{Covariates}

We considered participants' age, race, education, marital status,poverty-to-income ratio (PIR) $\square$ body mass index (BMI), physical activity, alcohol consumption, total energy intake and physical conditions (diabetes, kidney disease) as potential confounders. Participants chewing tobacco or using nicotine replacement therapy were excluded because these behaviors could disturb the quantification of the biomarkers ${ }^{24}$.

\section{Statistical analysis}

We tested the normality of continuous variables by Kolmogorov-Smirnov normality tests and described normal distributed variables with mean \pm standard deviation, non-normal distributed variables with median (interquartile range). We adopted Student's t-tests to compare the mean levels between the hypertension group and the non-hypertension group if the variable was normally distributed, otherwise, the Mann-Whitney $U$ test was adopted. And chi-square tests were used to compare the percentages of categorical variables between different groups.

For the current study, serum 25(OH)D was categorized into three groups $(<50 \mathrm{nmol} / \mathrm{L}, 50-75 \mathrm{nmol} / \mathrm{L}$ or $\geq 75$ $\mathrm{nmol} / \mathrm{L}$ ) based on the criteria set by IOM, and the highest level was the reference category; and the participants were categorized into two groups (yes or no) according to exposure to secondhand smoke, and the non-SHS was the reference category. Logistic regression models were performed to describe the association between exposure to SHS and vitamin D on the prevalence of hypertension and odds ratios [OR, with $95 \%$ confidence intervals $(\mathrm{Cl})$ ] were used to evaluate the risk associated with hypertension. In multivariate logistic regressions, model 1 adjusted for age, race, education level, marital status, PIR, and model 2 further adjusted for BMI, alcohol use, physical activity, diabetes, kidney disease. We examined interaction on the multiplicative scale. For multiplicative interaction, we calculated two-sided $P$ values to assess the significance of each product term in the logistic regression models and compared the ORs for SHS and hypertension across strata of serum 25(OH)D. And in order to further clarify the association, the stratified analysis by BMI and race then performed to determine the joint effect of vitamin D and exposure to SHS on hypertension in different groups. All statistical analyses were performed by using SPSS 22.0 software.

\section{Results}

A total of 2802 non-smoking childbearing-age women were included in the present study. In this population, the prevalence of hypertension was $19.8 \%$. The baseline characteristics of participants by hypertension were shown in the supplementary material. Individuals with hypertension had a higher rate of VDD than those without hypertension (48.2\% vs $40.7 \%, P=0.005)$, as well as the exposure rate of secondhand smoke (35.7\% vs $30.5 \%, P=0.017)$. Additionally, compared with participants without hypertension, those with hypertension tended to be older, obese, had a lower educational level and a lower level of physical activity $(P<0.05)$. 
Table 1 shows the effects of serum 25(OH)D and secondhand smoke on hypertension, respectively. In binary logistic regression analyses, by comparison to the vitamin D sufficient group, the OR with $95 \% \mathrm{Cl}$ of hypertension for the vitamin $\mathrm{D}$ deficiency group was $1.39(1.09-1.77)(P=0.009)$. In model 1 , after adjusting for age, race, education level, marital status and PIR, serum 25(OH)D was still inversely associated with the risk of hypertension ( $\mathrm{aOR}=1.65,95 \% \mathrm{Cl} 1.22-2.25, P=0.001)$. Further adjusted $\mathrm{BMI}$, alcohol use, physical activity, diabetes and kidney disease in model 2 , the logistic regression analysis revealed the vitamin $D$ deficiency as an independent predictor of hypertension (aOR $=1.47,95 \% \mathrm{Cl} 1.03-2.08, P=0.035)$.

Table 1

Odds ratios (ORs, 95\% confidence intervals (Cls)) of hypertension according to serum $25(\mathrm{OH}) \mathrm{D}$ concentrations and SHS as categorical using a logistics regression model, NHANES 2007-2014( $\mathrm{N}=2802)$

\begin{tabular}{|c|c|c|c|c|c|c|}
\hline & Crude OR & p-value & Model 1 OR & p-value & Model 2 OR & p-value \\
\hline 25(OH)D level & $1.39(1.09,1.77)$ & 0.009 & $1.65(1.22,2.25)$ & 0.001 & $1.47(1.03,2.08)$ & 0.035 \\
\hline$₫ 50 \mathrm{nmol} / \mathrm{L}$ & $1.03(0.80-1.34)$ & 0.806 & $1.26(0.93,1.69)$ & 0.132 & $1.17(0.84,1.63)$ & 0.363 \\
\hline \multicolumn{7}{|l|}{$50-75 \mathrm{nmol} / \mathrm{L}$} \\
\hline$\geq 75 \mathrm{nmol} / \mathrm{L}$ & Ref. & - & Ref. & - & Ref. & - \\
\hline SHS & $1.27(1.04,1.54)$ & 0.017 & $1.12(0.89,1.41)$ & 0.346 & $1.10(0.85,1.43)$ & 0.453 \\
\hline Yes & Ref. & - & Ref. & - & Ref. & - \\
\hline \multicolumn{7}{|l|}{ No } \\
\hline \multicolumn{7}{|c|}{ Model 1: adjusted for age, race, education level, marital status, PIR } \\
\hline \multicolumn{7}{|c|}{ Model 2: model $1+\mathrm{BMI}$, alcohol use, physical activity, diabetes, kidney disease } \\
\hline SHS, secondha & d smoke & & & & & \\
\hline
\end{tabular}

The ORs with 95\% Cls of hypertension based on whether or not exposed to SHS are shown in Table 1. Compared to those who were not exposed to SHS, those who exposed to SHS had a crude OR $(95 \% \mathrm{Cl})$ of 1.27 (1.04-1.54) $(P=0.017)$ for hypertension. However, after adjusted according to models 1 and 2 , a positive association between SHS and the risk of hypertension disappeared. Next, we divided the whole population into 6 groups according to 25(OH)D level and whether they were exposed to secondhand smoke or not (group 1: Deficiency and SHS; 2: Insufficiency and SHS; 3: Sufficiency and SHS; 4: Deficiency and non-SHS; 5 : Insufficiency and non-SHS; 6: Sufficiency and non-SHS). As shown in Table 2, with sufficient 25(OH)D and no exposure to secondhand smoke (group 6) as a reference,the crude ORs with $95 \%$ Cls of hypertension for the other five groups in order were 1.91 (1.38-2.63), $0.98(0.66-1.47), 1.29(0.83-2.03), 1.26(0.93-1.71)$, and $1.16(0.85-1.58)$, respectively. Of all the results, only $25(\mathrm{OH}) \mathrm{D}$ deficiency and exposure to secondhand smoke (group 1) had statistical significance. After adjusting the confounding factors, it was still associated with hypertension. In Model 2, compared to those who "Sufficiency and non-SHS", those who "Deficiency and SHS" had a multivariate-adjusted OR $(95 \% \mathrm{Cl})$ of $1.83(1.17-2.87)(P=0.009)$. The effect of the interaction between $25(\mathrm{OH}) \mathrm{D}$ and SHS on hypertension after adjusting for confounding factors (model 2) is shown in Fig. 2. 
Table 2

Interaction analysis of SHS and 25(OH)D on the risk of hypertension

\begin{tabular}{|c|c|c|c|c|c|c|}
\hline Interaction & $\begin{array}{l}\text { Crude OR, } \\
95 \% \mathrm{Cl}\end{array}$ & $P$ & $\begin{array}{l}\text { Model } 1 \text { OR, } \\
95 \% \mathrm{Cl}\end{array}$ & $P$ & $\begin{array}{l}\text { Model } 2 \text { OR, } \\
95 \% \mathrm{Cl}\end{array}$ & $P$ \\
\hline Deficiency*SHS & $1.91(1.38,2.63)$ & 0.000 & $2.04(1.38,3.03)$ & 0.000 & $1.83(1.17,2.87)$ & 0.009 \\
\hline Insufficiency*SHS & $0.98(0.66,1.47)$ & 0.933 & $1.09(0.70,1.71)$ & 0.697 & $0.99(0.60,1.64)$ & 0.974 \\
\hline Sufficiency*SHS & $1.29(0.83,2.02)$ & 0.263 & $1.25(0.75,2.09)$ & 0.390 & $1.18(0.66,2.09)$ & 0.578 \\
\hline Deficiency*non-SHS & $1.26(0.93,1.71)$ & 0.129 & $1.60(1.12,2.28)$ & 0.011 & $1.37(0.91,2.05)$ & 0.131 \\
\hline $\begin{array}{l}\text { Insufficiency*non- } \\
\text { SHS }\end{array}$ & $1.16(0.85,1.58)$ & 0.344 & $1.43(1.02,2.02)$ & 0.040 & $1.31(0.90,1.93)$ & 0.163 \\
\hline Sufficiency*non-SHS & Ref. & - & Ref. & - & Ref. & - \\
\hline \multicolumn{7}{|c|}{ Model 1: age, race, education level, marital status, PIR } \\
\hline \multicolumn{7}{|c|}{ Model 2: model $1+\mathrm{BMI}$, alcohol use, physical activity, diabetes, kidney disease } \\
\hline SH & & & & & & \\
\hline
\end{tabular}

Stratified analyses by BMI, the association of 25(OH)D and the risk of hypertension was only significant in the overweight group $\left(\mathrm{BMI}=25-30 \mathrm{~kg} / \mathrm{m}^{2}\right)$. Likewise, a significant interaction between SHS and 25(OH)D was found for the overweight group only. The effects of 25(OH)D and SHS alone and their interaction on hypertension in overweight are shown in Fig. 3. Among the overweight group, the aORs with $95 \% \mathrm{Cls}$ of hypertension were $2.37(1.03-2.47)(P=0.043)$, and $1.47(0.85-2.55)(P=0.173)$ in model 2 for $25(\mathrm{OH}) \mathrm{D}$ deficiency and exposure to SHS, respectively. Compared with the "Sufficiency and non-SHS" group,the aOR $(95 \%)$ of the "deficiency and SHS" group was 4.14(1.48-11.58) $(P=0.007)$.

After stratification by race, the above results were statistically significant among non-Hispanic black. In the non-Hispanic black stratification, compared with the sufficient group, the aOR $(95 \% \mathrm{Cl})$ of the effect of $25(\mathrm{OH}) \mathrm{D}$ deficiency on hypertension was $2.74(1.11-6.73)(P=0.028)$. Compared with the "Sufficiency and non-SHS" group,the aOR $(95 \% \mathrm{Cl})$ of the "deficiency and SHS" group was $4.60(1.53-13.80)(P=0.006)$. There was no significance among other races. The results as shown in Fig. 4.

\section{Discussion}

We found a joint effect of vitamin D deficiency and exposure to secondhand smoke on the prevalence of hypertension in American women of childbearing age, and the synergistic effect was more significant in overweight and Non-Hispanic Black women.

Many studies have supported the relationship between low serum 25(OH)D levels and a high risk of hypertension. In a 14-year follow-up cohort of Caucasian women, they suggested that vitamin D insufficiency was associated with 3 fold increased risk of systolic hypertension after adjusting for age, body fat percentage, antihypertensive medication use, and smoking ${ }^{29}$. Meanwhile, in the research of Forman et al.,compared with 
the highest quartile of serum 25(OH)D, women in the lowest had an adjusted odds ratio for incident hypertension of $1.66(95 \% \mathrm{Cl}: 1.11 \text { to } 2.48 ; P=0.01)^{10}$. Another cross-sectional study of 2098 premenopausal and 2298 postmenopausal women showed that serum 25(OH)D concentrations were associated with a lower risk of hypertension in premenopausal women ${ }^{12}$. However, there are still a few inconsistencies in research conclusions. A meta-analysis ${ }^{30}$ included seven prospective studies for 53,375 participants shows that lower serum 25(OH)D concentrations were not associated with a greater risk of incident hypertension. Another metaanalysis identified 11 cohort studies and 27 RCTs, with 43,320 and 3,810 participants, respectively ${ }^{31}$. The results indicate that supplementation with vitamin $D$ does not lower blood pressure in the general population. Our results are in line with observational and experimental evidence linking lower serum 25(OH)D levels in women of childbearing age with hypertension and further provide a theoretical basis.

Few previous studies have explored the effects of passive smoking on hypertension in women of childbearing age, although the correlation between active smoking and hypertension has been clearly established ${ }^{32,33}$. A Bulgarian study involving former smokers and nonsmokers found that passive smoking was not significantly associated with hypertension ${ }^{34}$. However,several studies in Asian populations reported that exposure to SHS was associated with the risk of high blood pressure. In rural Chinese nonsmoking female (age range: 33-82 years) population, Li Nan et al. found that passive smoking was associated with a higher risk of hypertension ${ }^{35}$. One prospective cohort study in 106268 Korean discovered in Korea evaluated the association between exposure to SHS in self-reported never-smokers verified by urinary cotinine and hypertension. ${ }^{36}$ They observed that Current SHS exposure that has been exposed to home SHS $(1.22(1.11,1.33))$, as well as current SHS exposure only at the workplace $(1.15(1.02,1.29))$, significantly increased the ORs for hypertension compared to no SHS exposure. Similar relationships have been found in children and adolescents. The effects of secondhand smoke exposure on the blood pressure of 3579 children and adolescents aged 8 to 17 were analyzed using NHANES from 2007 to 2012. The results showed that children exposed to high levels of secondhand smoke were 1.966 (95\% Cl: 1.31-2.951) times more likely to be in the range of hypertension than those without secondhand smoke exposure ${ }^{37}$. A large study, conducted among a sample of 42745 children ages 7-18 years ${ }^{38}$, observed that parental self-report of household smoking was associated with childhood hypertension (adjusted OR $=1.11,95 \% \mathrm{Cl}: 1.02,1.20$ ). No significant associations were found in boys. Similarly, among adults ( $\geq 18$ years) in the United States of America, SHS was associated with increased odds of hypertension among women (aOR: $1.240,95 \% \mathrm{Cl}: 1.238$ to 1.242 ), $P<0.0001$ but not among men (aOR: $0.755,95 \% \mathrm{Cl}: 0.754$ to 0.757 ), $P<0.0001$. However, this study used self-reported information to evaluate whether exposure to SHS. In our study, exposure to SHS was associated with greater hypertension risk, but the association became non-significant after adjusting for potential confounders. The results were contrary to our expectations; it may be due to the research design, the selection of the population, and the criteria for judging secondhand smoke exposure are different. Further research is worth being conducted on the hypothesis that exposure to secondhand smoke increases the risk of hypertension in women of childbearing age.

Our epidemiological data indicate that SHS represents an important environmental factor contributing to hypertension in females with VDD. The possible underlying mechanisms are as follows. On one hand, studies have shown that smoking may trigger relevant potential pathways disrupting VD endocrine system (VDES) leading to VDD. The main ways are: first, smoking inhibits the expression of CYP27B1(the key enzyme 
required for activation of $\mathrm{VD}^{39}$ ), decreases the level of serum parathyroid hormone $(\mathrm{PTH})$, increases the exposure to cadmium and lead, and increases the expression of CYP24A1(the key enzyme required for 24-hydroxylase activity) to reduce the levels of $25(\mathrm{OH}) \mathrm{D}$ and $25(\mathrm{OH})_{2} \mathrm{D}$ in serum; second, smoking inhibits the intake of VD, from diet ${ }^{14}$; third, smoking promotes skin aging and thus hinders the synthesis of VD ${ }^{14}$. On the other hand, the effect of this ability of vitamin $D$ to negatively regulate the renin-angiotensin system (RAS) and the association with endothelial vasodilator dysfunction ${ }^{40-42}$. Passive smoking may have adverse effects on vasoconstriction and/or vasodilation, due to nicotine causes vasoconstriction, which results in transient increases in BP. Further, Passive smoking may also lead to vascular endothelial dysfunction by affecting vascular endothelial cells ${ }^{43,44}$. Zhang $\mathrm{J}$ et. $\mathrm{al}^{45}$ posited the endothelial dysfunction was mediated as a result of systemic inflammation. The inflammatory responses and oxidative stress responses due to vitamin $D$ deficiency may be aggravated by SHS. This may aggravate the effect on vascular endothelial function and lead to hypertension when both vitamin D deficiency and secondhand smoke exposure, were present. Our results are consistent with a number of previous studies evaluating the independent associations between vitamin D and hypertension, and our identification of statistical interaction with secondhand smoke exposure may support the hypothesized biological mechanisms of these associations.

Overweight enhances associations between the interaction between VDD and SHS on hypertension. The finding that overweight may increase susceptibility to VDD has potential clinical and public health relevance. Potential pathways may involve the effects of overweight and SHS exposure on the interdependence of airway and systemic oxidative stress and inflammation ${ }^{46}$, as well as their effects on VD deficiency ${ }^{47}$. However, we did not find the interaction in the obesity group. Our conjecture remains to be further confirmed.

There are several strengths in our study. First, hypertension was defined by using the new diagnostic criteria for hypertension in 2017. The new guidelines lower the standard of high blood pressure and will require more adults to receive antihypertensive treatment. And it can prevent about 610000 CVD events and 334000 deaths each year in the United States alone ${ }^{48}$, and reduce the resulting economic burden. Second, to our knowledge, this is the first study to explore the associations between the combined exposures of vitamin D deficiency and SHS and the risk of hypertension among the general American women of childbearing age. Third, we used the objective index of serum cotinine to classify whether exposed to SHS or not, and the result is more reliable. Previous studies may be limited by exposure assessment because self-report of exposure to SHS may not be as accurate as biological markers of exposure. The use of biomarkers could reduce measurement error. Cotinine is the principal metabolite of nicotine and can be measured in serum, urine, or saliva; it has been effectively used as a sensitive biomarker for exposure to tobacco in both active and SHS due to its long halflife ${ }^{49}$.

However, there are still several limitations in our study. First, because of the cross-sectional design of the study, it is difficult to determine causality. Second, according to the information of the questionnaire, women who have self-reported active smoking were excluded, and this method is often imprecise because of conscious or unconscious mis-recordings and under-reporting and there might be ineluctable recall bias. Third, we cannot rule out all the possible residual confound factors from unmeasured confounders.

\section{Conclusion}


In summary, vitamin D deficiency was associated with a higher risk of hypertension, and the joint effect of exposure to secondhand smoke was higher than the risks associated with each individual factor in women of childbearing age. These findings add new theoretical support for the potential risk of hypertension and reinforce the view that low 25(OH)D and secondhand smoke exposure are risk factors for hypertension. The findings may facilitate planning and guiding targeted strategies, which may include a total ban or more stringent restrictions on smoking, as well as population VD supplements to prevent and control high blood pressure in women of childbearing age around the world. In addition, our study highlights the need for future prospective studies and mechanism research to further explore the burden and impact of secondhand smoke exposure and vitamin D deficiency on the outcomes of women of childbearing age or pregnant women and fetuses.

\section{Declarations}

\section{Ethics approval and consent to participate}

The NCHS Research Ethics Review Board reviewed and approved the study, and informed written consent was obtained from all participants before they took part in the study.

\section{Consent for publication}

Not applicable.

\section{Availability of data and materials}

Data are available at NHANES - National Health and Nutrition Examination Survey Homepage (cdc.gov). Further database is available on request.

\section{Competing interests}

The authors declared that they have no competing interests.

\section{Funding}

We thank the Zhendong Foundation of the Chinese Nutrition Society (CNS, No. CNS2019079B-5) for its support. The findings achieved herein are solely the responsibility of the authors.

\section{Authors' contributions}

Q.Q. Shen and Q. Xu conceived the study, collected and organized data, carried out the statistical analysis, and drafted the manuscript. G.J. Li participated in the study design, in the coordination and the execution of data collection, statistical analysis and in writing the manuscript. Others contributed to the data management, and gave critical appraisal of the manuscript. Q. Z. Wang coordinated the study design, and gave critical appraisal of the manuscript. All authors read and approved the final manuscript.

\section{Acknowledgements}


The authors would like to thank the study participants and all staff members who were involved in conducting the study.

\section{Authors' information}

${ }^{1}$ Public Health School, Medical College of Qingdao University, Qingdao, China. ${ }^{2}$ Qingdao Women and Children's Hospital, Qingdao University, Qingdao, China. ${ }^{3}$ The Affiliated Hospital of Qingdao University, Qingdao, China

\section{References}

1. Song JJ, Ma Z, Wang J, Chen LX, Zhong JC: Gender Differences in Hypertension. Journal of cardiovascular translational research 2020, 13(1):47-54.

2. Mills KT, Stefanescu A, He J: The global epidemiology of hypertension. Nature reviews Nephrology 2020, 16(4):223-237.

3. Forouzanfar MH, Liu P, Roth GA, Ng M, Biryukov S, Marczak L, Alexander L, Estep K, Hassen Abate K, Akinyemiju TF et al: Global Burden of Hypertension and Systolic Blood Pressure of at Least 110 to $115 \mathrm{~mm}$ Hg, 1990-2015. JAMA 2017, 317(2):165-182.

4. Liu X, Olsen J, Agerbo E, Yuan W, Wu CS, Li J: Maternal preeclampsia and childhood asthma in the offspring. Pediatric allergy and immunology : official publication of the European Society of Pediatric Allergy and Immunology 2015, 26(2):181-185.

5. Zhang M, Michos ED, Wang G, Wang X, Mueller NT: Associations of Cord Blood Vitamin D and Preeclampsia With Offspring Blood Pressure in Childhood and Adolescence. JAMA Netw Open 2020, 3(10):e2019046.

6. Xu X, Ritz B, Cockburn M, Lombardi C, Heck JE: Maternal Preeclampsia and Odds of Childhood Cancers in Offspring: A California Statewide Case-Control Study. Paediatric and perinatal epidemiology 2017, 31(2):157-164.

7. Brahmbhatt Y, Gupta M, Hamrahian S: Hypertension in Premenopausal and Postmenopausal Women. Current hypertension reports 2019, 21(10):74.

8. Whelton PK, Carey RM, Aronow WS, Casey DE, Jr., Collins KJ, Dennison Himmelfarb C, DePalma SM, Gidding S, Jamerson KA, Jones DW et al: 2017 ACC/AHAVAAPAABC/ACPM/AGS/APhA/ASH/ASPC/NMA/PCNA Guideline for the Prevention, Detection, Evaluation, and Management of High Blood Pressure in Adults: A Report of the American College of Cardiology/American Heart Association Task Force on Clinical Practice Guidelines. Journal of the American College of Cardiology 2018, 71(19):e127-e248.

9. Roth DE, Abrams SA, Aloia J, Bergeron G, Bourassa MW, Brown KH, Calvo MS, Cashman KD, Combs G, De-Regil LM et al: Global prevalence and disease burden of vitamin D deficiency: a roadmap for action in 
low- and middle-income countries. Annals of the New York Academy of Sciences 2018, 1430(1):44-79.

10. Forman JP, Curhan GC, Taylor EN: Plasma 25-hydroxyvitamin D levels and risk of incident hypertension among young women. Hypertension (Dallas, Tex : 1979) 2008, 52(5):828-832.

11. Vishnu A, Ahuja V: Vitamin D and Blood Pressure Among U.S. Adults: A Cross-sectional Examination by Race/Ethnicity and Gender. American journal of preventive medicine 2017, 53(5):670-679.

12. Kwak JH, Hong YC, Choi YH: Serum 25-hydroxyvitamin D and hypertension in premenopausal and postmenopausal women: National Health and Nutrition Examination Surveys 2007-2010. Public health nutrition 2020, 23(7):1236-1246.

13. Qi D, Nie X, Wu S, Cai JJPo: Vitamin D and hypertension: Prospective study and meta-analysis. 2017, 12(3):e0174298.

14. Mousavi SE, Amini H, Heydarpour P, Amini Chermahini F, Godderis L: Air pollution, environmental chemicals, and smoking may trigger vitamin $\mathrm{D}$ deficiency: Evidence and potential mechanisms. Environment international 2019, 122:67-90.

15. Current Cigarette Smoking Among Adults in the United

States [https://www.cdc.gov/tobacco/data_statistics/fact_sheets/adult_data/cig_smoking/index.htm\#nation]

16. Tsai J, Homa DM, Gentzke AS, Mahoney M, Sharapova SR, Sosnoff CS, Caron KT, Wang L, Melstrom PC, Trivers KF: Exposure to Secondhand Smoke Among Nonsmokers - United States, 1988-2014. MMWR Morbidity and mortality weekly report 2018, 67(48):1342-1346.

17. Alshaarawy 0 , Xiao J, Shankar A: Association of serum cotinine levels and hypertension in never smokers. Hypertension (Dallas, Tex : 1979) 2013, 61(2):304-308.

18. Park YS, Lee CH, Kim YI, Ahn CM, Kim JO, Park JH, Lee SH, Kim JY, Chun EM, Jung TH et al: Association between secondhand smoke exposure and hypertension in never smokers: a cross-sectional survey using data from Korean National Health and Nutritional Examination Survey V, 2010-2012. BMJ open 2018, 8(5):e021217.

19. Skipina TM, Soliman EZ, Upadhya B: Association between secondhand smoke exposure and hypertension: nearly as large as smoking. Journal of hypertension 2020, 38(10):1899-1908.

20. Latic N, Erben RG: Vitamin D and Cardiovascular Disease, with Emphasis on Hypertension, Atherosclerosis, and Heart Failure. International journal of molecular sciences 2020, 21(18).

21. Analytical Note for 25-Hydroxyvitamin D Data Analysis using NHANES III (1988-1994), NHANES 2001-2006, and NHANES 2007-2010 (October 2015) [https://wwwn.cdc.gov/Nchs/Nhanes/vitamind/analyticalnote.aspx?h=/Nchs/Nhanes/20132014/VID_H.htm\&t=VID_H\%20Doc] 
22. Holick MF, Binkley NC, Bischoff-Ferrari HA, Gordon CM, Hanley DA, Heaney RP, Murad MH, Weaver $\mathrm{CM}$, Endocrine S: Evaluation, treatment, and prevention of vitamin D deficiency: an Endocrine Society clinical practice guideline. The Journal of clinical endocrinology and metabolism 2011, 96(7):1911-1930.

23. Bagchi P, Geldner N, deCastro BR, De Jesus VR, Park SK, Blount BC: Crotonaldehyde exposure in U.S. tobacco smokers and nonsmokers: NHANES 2005-2006 and 2011-2012. Environmental research 2018, 163:19 .

24. Fu Z, Jiang H, Xu Z, Li H, Wu N, Yin P: Objective secondhand smoke exposure in chronic obstructive pulmonary disease patients without active smoking: the U.S. National Health and Nutrition Examination Survey (NHANES) 2007-2012. Annals of translational medicine 2020, 8(7):445.

25. Espenship MF, Silva LK, Smith MM, Capella KM, Reese CM, Rasio JP, Woodford AM, Geldner NB, Rey deCastro B, De Jesús VR et al: Nitromethane Exposure from Tobacco Smoke and Diet in the U.S. Population: NHANES, 2007-2012. Environmental science \& technology 2019, 53(4):2134-2140.

26. Homa D, Neff L, King B, Caraballo R, Bunnell R, Babb S, Garrett B, Sosnoff C, Wang L, JMM et al: Vital signs: disparities in nonsmokers' exposure to secondhand smoke-United States, 1999-2012. 2015, 64(4):103108.

27. Gan WQ, Mannino DM, Jemal A: Socioeconomic disparities in secondhand smoke exposure among US never-smoking adults: the National Health and Nutrition Examination Survey 1988-2010. Tobacco control 2015, 24(6):568-573.

28. Pérez-Stable E, Herrera B, Jacob P, Benowitz NJJ: Nicotine metabolism and intake in black and white smokers. 1998, 280(2):152-156.

29. Griffin FC, Gadegbeku CA, Sowers MR: Vitamin D and subsequent systolic hypertension among women. American journal of hypertension 2011, 24(3):316-321.

30. Qi D, Nie XL, Wu S, Cai J: Vitamin D and hypertension: Prospective study and meta-analysis. PloS one 2017, 12(3):e0174298.

31. Zhang D, Cheng C, Wang Y, Sun H, Yu S, Xue Y, Liu Y, Li W, Li X: Effect of Vitamin D on Blood Pressure and Hypertension in the General Population: An Update Meta-Analysis of Cohort Studies and Randomized Controlled Trials. Preventing chronic disease 2020, 17:E03.

32. Feng D, Liu T, Su DF, Wang H, Ding P, He YH, Deng XQ, Hou MJ, Ling WH, Chen WQ: The association between smoking quantity and hypertension mediated by inflammation in Chinese current smokers. Journal of hypertension 2013, 31(9):1798-1805.

33. Gao K, Shi X, Wang WJSr: The life-course impact of smoking on hypertension, myocardial infarction and respiratory diseases. 2017, 7(1):4330.

34. Sipetić Grujičić S, Terzić Supić Z, Nikolić Ž, Gredić D, Bjekić M, Bjegović V, Ratkov IJMgopotMAoZ-DC, Bosnia, Herzegovina: Risk factors for the development of arterial hypertension. 2014, 11(1):19-25. 
35. Li N, Li Z, Chen S, Yang N, Ren A, Ye R: Effects of passive smoking on hypertension in rural Chinese nonsmoking women. Journal of hypertension 2015, 33(11):2210-2214.

36. Kim BJ, Kang JG, Kim JH, Seo DC, Sung KC, Kim BS, Kang JH: Association between Secondhand Smoke Exposure and Hypertension in 106,268 Korean Self-Reported Never-Smokers Verified by Cotinine. Journal of clinical medicine 2019, 8(8).

37. Liu SH, Liu B, Sanders AP, Saland J, Wilson KM: Secondhand smoke exposure and higher blood pressure in children and adolescents participating in NHANES. Prev Med 2020, 134:106052.

38. Zhang Z, Ma J, Wang Z, Dong Y, Yang Z, Dong B, Ma Y: Parental smoking and blood pressure in children and adolescents: a national cross-sectional study in China. BMC pediatrics 2019, 19(1):116.

39. Cheng J, Levine M, Bell N, Mangelsdorf D, Russell DJPotNAoSotUSoA: Genetic evidence that the human CYP2R1 enzyme is a key vitamin D 25-hydroxylase. 2004, 101(20):7711-7715.

40. Li YC: Vitamin D regulation of the renin-angiotensin system. Journal of cellular biochemistry 2003, 88(2):327-331.

41. Tare M, Emmett SJ, Coleman HA, Skordilis C, Eyles DW, Morley R, Parkington HC: Vitamin D insufficiency is associated with impaired vascular endothelial and smooth muscle function and hypertension in young rats. The Journal of physiology 2011, 589(Pt 19):4777-4786.

42. Del Pinto R, Ferri C: Inflammation-Accelerated Senescence and the Cardiovascular System: Mechanisms and Perspectives. International journal of molecular sciences 2018, 19(12).

43. Celermajer D, Adams M, Clarkson P, Robinson J, McCredie R, Donald A, Deanfield JJTNEjom: Passive smoking and impaired endothelium-dependent arterial dilatation in healthy young adults. 1996, 334(3):150154.

44. Heiss C, Amabile N, Lee A, Real W, Schick S, Lao D, Wong M, Jahn S, Angeli F, Minasi P et al: Brief secondhand smoke exposure depresses endothelial progenitor cells activity and endothelial function: sustained vascular injury and blunted nitric oxide production. 2008, 51(18):1760-1771.

45. JIN ZHANG YL, JIAQI SHI,t DOUGLAS F. LARSON,t AND;RONALD Ross WATSON: Side-stream cigarette smoke induces dose-response in systemic inflammatory cytokine production and oxidative stress. Experimental Biology and Medicine 2002.

46. Wu TD, Brigham EP, Peng R, Koehler K, Rand C, Matsui EC, Diette GB, Hansel NN, McCormack MC: Overweight/obesity enhances associations between secondhand smoke exposure and asthma morbidity in children. The Journal of Allergy and Clinical Immunology: In Practice 2018, 6(6):2157-2159.e2155.

47. Pourshahidi LJTPotNS: Vitamin D and obesity: current perspectives and future directions. 2015, 74(2):115-124. 
48. Bundy JD, Mills KT, Chen J, Li C, Greenland P, He J: Estimating the Association of the 2017 and 2014 Hypertension Guidelines With Cardiovascular Events and Deaths in US Adults: An Analysis of National Data. JAMA cardiology 2018, 3(7):572-581.

49. Benowitz N, Hukkanen J, Jacob PJHoep: Nicotine chemistry, metabolism, kinetics and biomarkers. 2009(192):29-60.

\section{Figures}

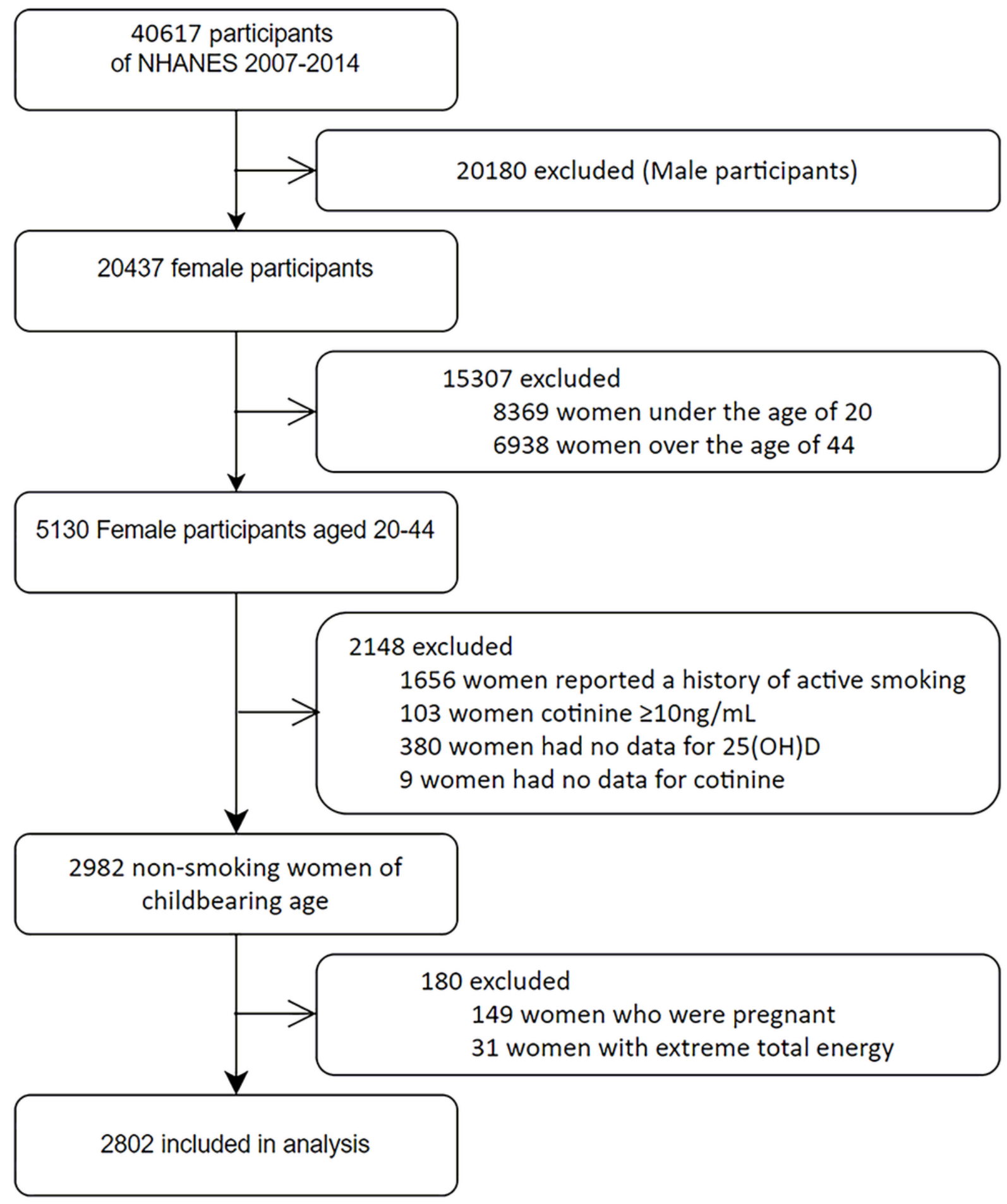


Figure 1

The flow of participant inclusion is displayed

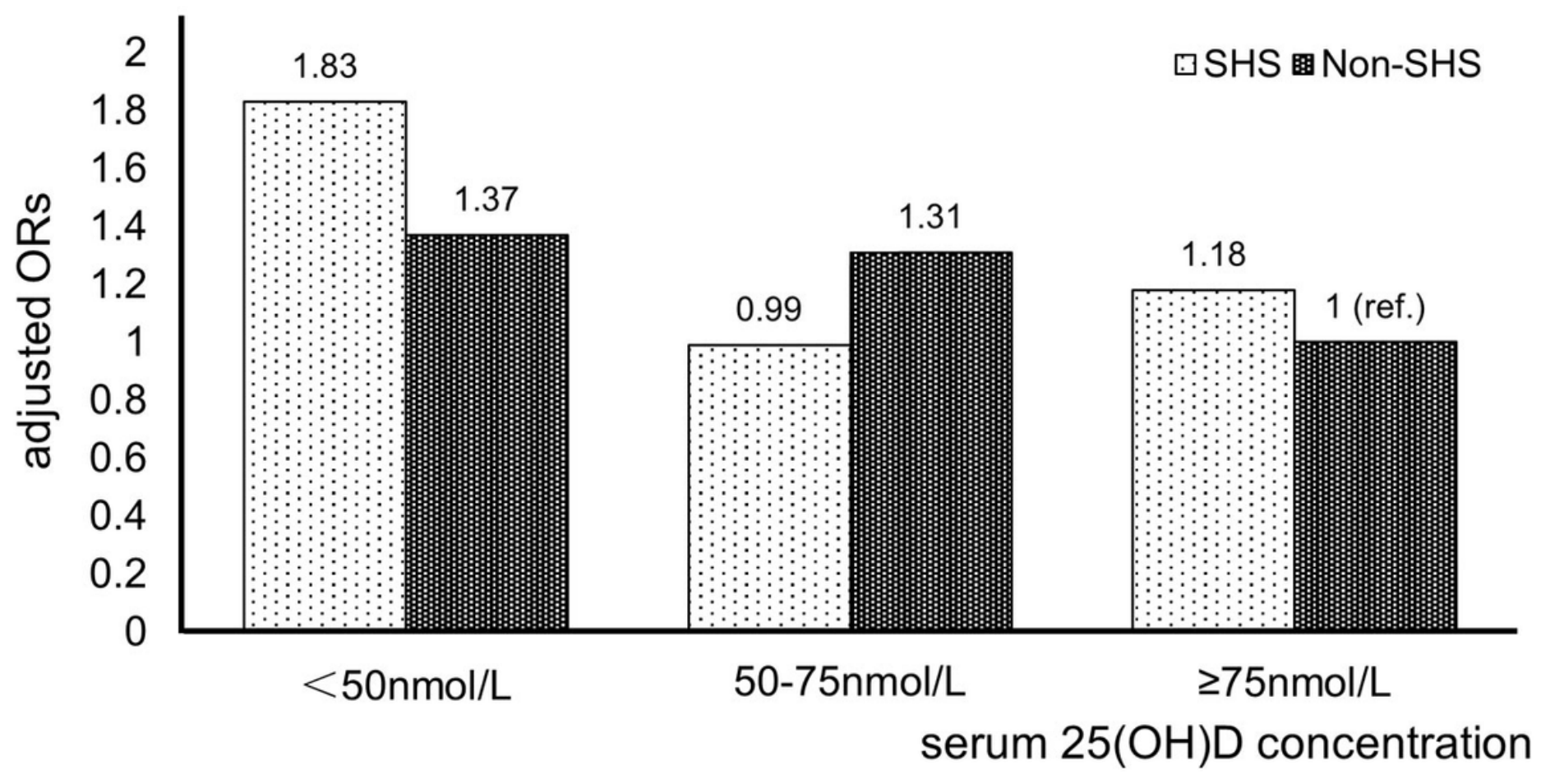

Figure 2

The effect of the interaction between 25(OH)D and SHS on hypertension after adjusting for confounding factors (model 2) 
Adjusted OR(95\% Cl)

$P$ value

$\nabla \quad 25(\mathrm{OH}) \mathrm{D}:<50 \mathrm{nmol} / \mathrm{L}^{1}$

च 25(OH)D:50-75nmol// $\mathrm{L}^{1}$

A $\mathrm{SHS}^{2}$

$\boldsymbol{\nabla}<50 \mathrm{nmol} / \mathrm{L}^{*} \mathrm{SHS}^{3}$

- $50-75 \mathrm{nmol} / \mathrm{L}^{*} \mathrm{SHS}{ }^{3}$

- $\geqslant 75 \mathrm{nmol} / \mathrm{L}^{*} \mathrm{SHS}^{3}$

* $<50 \mathrm{nmol} / \mathrm{L}^{*}$ Non-SHS ${ }^{3}$

$\diamond \quad 50-75 \mathrm{nmol} / \mathrm{L}^{*} \mathrm{Non}-\mathrm{SHS}^{3}$

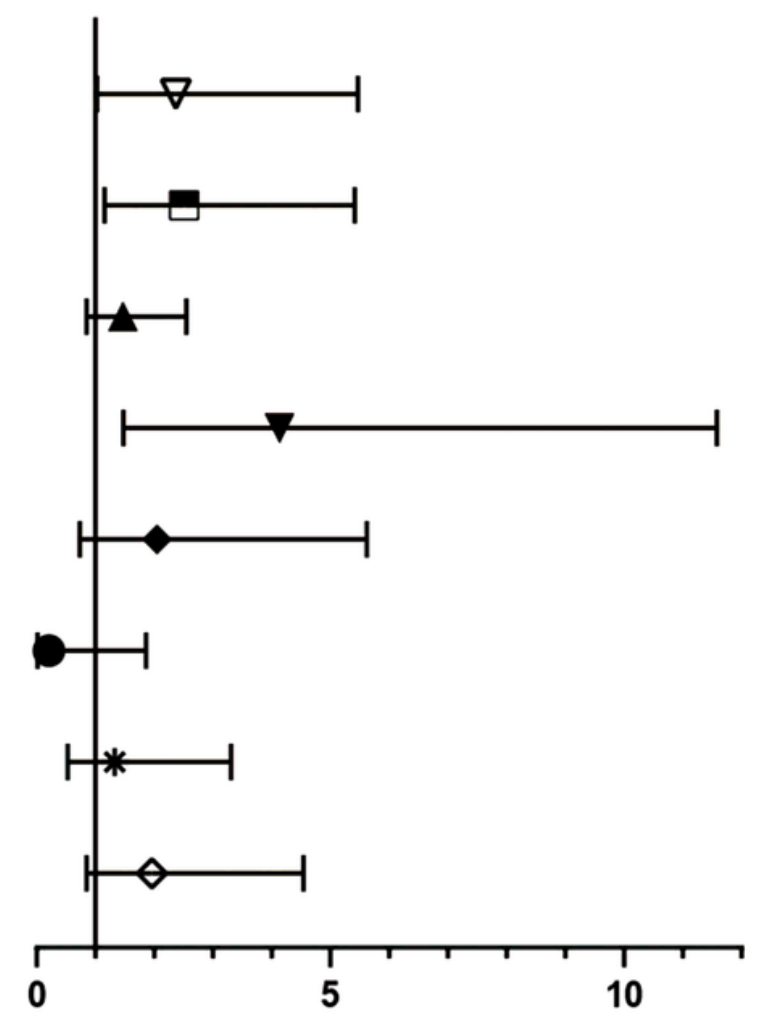

$2.37(1.03,5.47)$

$2.51(1.16,5.42)$

$1.47(0.85,2.55)$

$4.14(1.48,11.58)$

$2.05(0.74,5.62)$

$0.21(0.02,1.86)$

$1.33(0.53,3.31)$

$1.96(0.85,4.54)$
0.043

0.019

0.173

0.007

0.165

0.160

0.540

0.115

Figure 3

The effects of 25(OH)D and SHS alone and their interaction on hypertension in overweight 
Adjusted $\mathrm{OR}(95 \% \mathrm{Cl})$

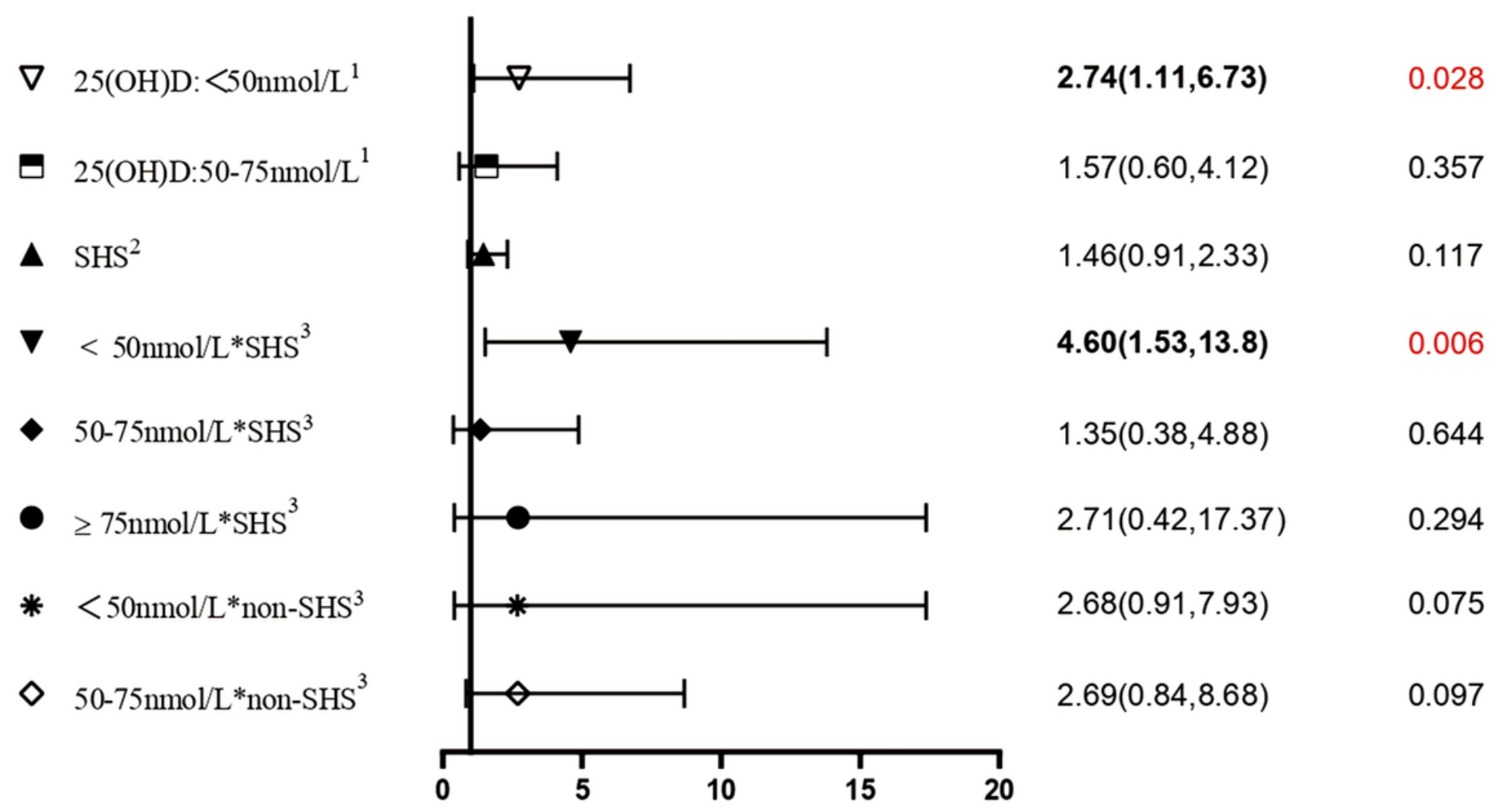

Figure 4

The aOR $(95 \% \mathrm{Cl})$ of the "deficiency and SHS" group was $4.60(1.53-13.80)(P=0.006)$. There was no significance among other races.

\section{Supplementary Files}

This is a list of supplementary files associated with this preprint. Click to download.

- supplementmaterial.docx 